\title{
Squeezing in Lithium Niobate Waveguides
}

F. Lenzini ${ }^{1,2}$, J. Janousek ${ }^{3,4}$, O. Thearle ${ }^{3,4}$, M. Villa ${ }^{1}$, B. Haylock ${ }^{1}$, S. Kasture ${ }^{1}$, L. Cui ${ }^{5}$, H.-P. Phan ${ }^{6,7}$, D. V. Dao ${ }^{6,7}$, H. Yonezawa ${ }^{8}$, P. K. Lam ${ }^{4}$, E. H. Huntington ${ }^{3}$, and M. Lobino ${ }^{1,6, *}$

${ }^{1}$ Centre for Quantum Computation \& Communication Technology and Centre for Quantum Dynamics, Griffith University, Brisbane QLD 4111, Australia; ${ }^{2}$ Institut of Physics, University of Muenster, 48149 Muenster, Germany; ${ }^{3}$ Centre for Quantum Computation \& Communication

Technology and Research School of Engineering, The Australian National University, Canberra ACT 2601, Australia; ${ }^{4}$ Centre for Quantum Computation \& Communication Technology and Department of Quantum Science, Research School of Physics and Engineering, The Australian National University, Canberra ACT 2601, Australia; ${ }^{5}$ College of Precision Instrument and Optoelectronics Engineering, Tianjin University, Tianjin 300072, China; ${ }^{6}$ Queensland Micro- and Nanotechnology Centre, Griffith University, Brisbane QLD 4111, Australia; ${ }^{7}$ School of Engineering, Griffith University, Brisbane QLD 4111, Australia; ${ }^{8}$ Centre for Quantum Computation \& Communication Technology and School of Engineering and Information Technology, The University of New South Wales, Canberra, ACT 2600, Australia

\begin{abstract}
Lithium niobate is widely used in photonics applications because of its large second order nonlinearity and the possibility of integrating nonlinear parametric processes and fast electro-optical reconfigurability. Here we show how these properties can be used for continuous variable quantum optics experiments including the generation, manipulation and characterization and detection of squeezed light.
\end{abstract}

Keywords: Integrated optics, quantum optics, squeezed light, lithium niobate

\section{INTRODUCTION}

Integrated quantum photonics has emerged as a promising platform for large scale optical quantum technology ${ }^{1}$. Confining light inside miniaturized waveguide circuits enables the generation, manipulation, and detection of quantum photonics states in a scalable way ${ }^{2}$. In particular lithium niobate has a great potential for realizing all basic operations in a single platform. Here we show the generation, manipulation, and the interferometric stage of homodyne detection of non-classical light on a single device, a key step towards a fully integrated approach to optical quantum technology with continuous variables ${ }^{3}$. Furthermore, the lithium niobate platform can be used for classical characterization of squeezing and the generation of ultrashort squeezing pulses which are essential for time multiplexed cluster state quantum computation ${ }^{8}$.

*m.lobino@griffith.edu.au; phone 6173 7354115; iqtlab.net

AOS Australian Conference on Optical Fibre Technology (ACOFT) and Australian Conference on Optics,

Lasers, and Spectroscopy (ACOLS) 2019, edited by Arnan Mitchell, Halina Rubinsztein-Dunlop, Proc. of SPIE

Vol. 11200, 1120012 - (C) 2019 SPIE · CCC code: 0277-786X/19/\$21 · doi: 10.1117/12.2539899 


\section{THE LITHIUM NIOBATE PLATFORM}

We fabricated a device to realize a reconfigurable lithium niobate waveguide network (see Fig. 1). The goal was to demonstrate the potential of this platform in generating and manipulating multiple quantum states. The sources of squeezed vacuum are two nonlinear periodically poled waveguides are pumped by a $777 \mathrm{~nm}$ laser, generating photons at $1554 \mathrm{~nm}$ through spontaneous parametric down conversion, and the two modes interfere in an electro-optically reconfigurable directional coupler. Two integrated frequency filters separate the pump from the signal while the output modes are characterized by two integrated homodyne detectors where signal and local oscillator (LO) are mixed and the phase of the LOs are scanned. Free space photodiodes are used for the measurements since they provide a detection efficiency $>99 \%$. This device can generate and characterizing squeezed vacuum and two-mode entangled states, essential resources for several optical quantum computation and communication protocols. We measure a squeezing level of $-1.38 \pm 0.04 \mathrm{~dB}$ and demonstrate two-mode entanglement from quadrature correlations and by verifying an inseparability criterion $\mathrm{I}=0.77 \pm 0.02<1^{3}$. Our platform can implement all the processes required for optical quantum technology and its high nonlinearity and fast reconfigurability makes it ideal for the realization of quantum computation with time encoded continuous variable cluster states ${ }^{4}$. Future development of this technology will enable the integration of several quantum photonic applications, including fast photon routing and frequency conversion for hybrid quantum networks.

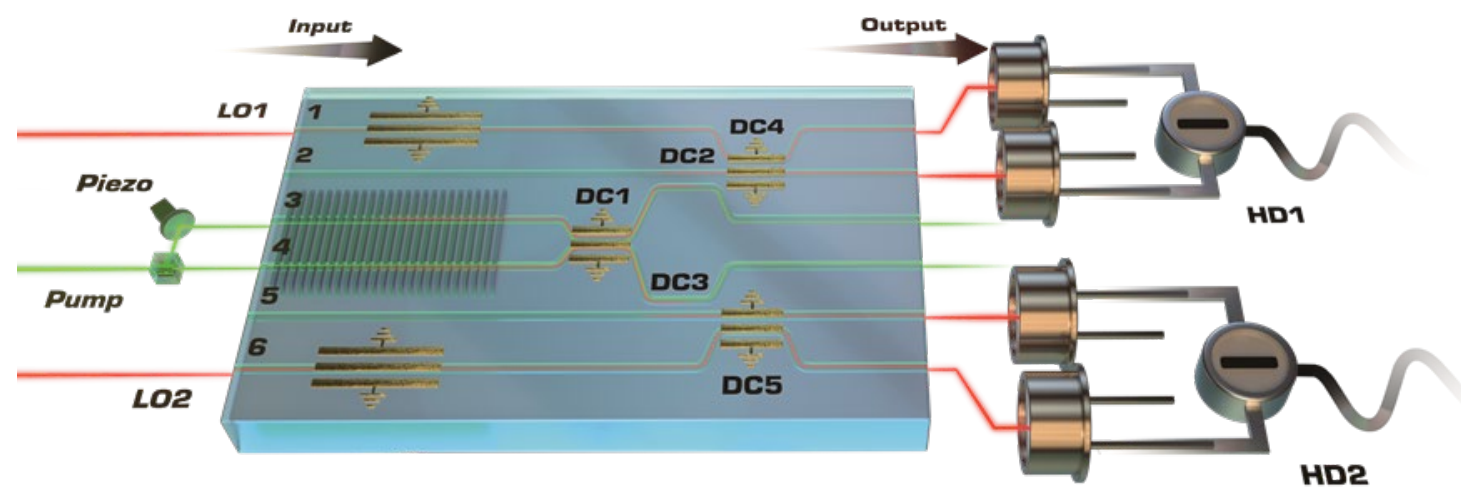

Figure 1. Schematic of the chip for the generation and manipulation of CV quantum states.

In separate experiments we characterized the bandwidth of the generated squeezed vacua using classical technique ${ }^{7}$. Finally we will report on the generation of ultrashort squeezed vacuum pulses using these integrated, broadband, single pass squeezers.

\section{REFERENCES}

[1] S. Tanzilli, et al., "On the genesis and evolution of Integrated Quantum Optics”, Laser Phot. Rev. 6, pp. 115 (2012).

[2] J. Carolan, "Universal linear optics", Science 349, pp. 711-716 (2015).

[3] W.P. Bowen, et al., "Experimental Investigation of Criteria for Continuous Variable Entanglement", Phys. Rev. Lett. 90, pp. 043601 (2003).

[4] F. Lenzini, et al., "Integrated photonic platform for quantum information with continuous variables", Science Advances 4, eaat9331 (2018).

[5] Lenzini et al., Active demultiplexing of single-photons from a solid-state source, Laser Photonics Rev. 11, 1600297 (2017).

[6] Kasture et al. Frequency conversion between UV and telecom wavelengths in a lithium niobate waveguide for quantum communication with $\mathrm{Yb}+$ trapped ions, J. Optics 18, 104007(2016).

[7] Lenzini et al, Direct characterization of a nonlinear photonic circuit's wave function with laser light, Light Sci. Appl. 7, 17143(2018). 
[8] N.C. Menicucci, “Temporal-mode continuous-variable cluster states using linear optics”, Phys. Rev. A 83, pp. 62314 (2011). 